\title{
Determining the cellular localization of C.elegans Editing Enzyme
}

\author{
Anna Dudley, Dr. Heather Hundley, Suba Rajendren \\ Indiana University School of Medicine, Department of Biochemistry \& Molecular \\ Biology, Jordon Hall, Indiana University, Bloomington Indiana.
}

\section{Background and Hypothesis:}

Over two thirds of human mRNAs contain adenosine(A)-to-inosine (I) editing sites indicating that RNA editing significantly alters the flow of genetic information. RNA editing is required for normal development and proper neuronal function in all animals. Aberrant RNA editing is identified in several neurological disorders and cancers.

A-to-I RNA editing is catalyzed by adenosine deaminases that act on RNA (ADARs) proteins. These enzymes commonly bind to double stranded RNA (dsRNA) and catalyze the conversion of adenosine to inosine. However, it is unknown whether these different regions of mRNA are edited by ADARs in the cytoplasm or nucleus. Early research has shown that inosines are present in the nucleus, while new additional evidence shows activity in cytoplasm as well. What dictates the location of the editing is yet unknown.

\section{Experimental Design:}

The subcellular localization of the ADAR editing enzyme will be determined by performing western blots of fractionated Caenorhabditis elegans embryos. Unlike mammals, ADAR editing is not essential in C. elegans, thus making it an easy system to address mechanistic questions about RNA editing. Embryos will be attained from wild-type, ADR-1 and ADR-2 knockout worms, and biochemical techniques will be used to obtain nuclei and cytoplasmic fractions. These fractions will be subjected to SDS-PAGE and western blotting for the ADAR editing enzyme, ADR-2. I will also use positive controls of a nuclear protein (histone) and a cytoplasmic protein (Tubulin) to test the fractionation.

\section{Anticipated Results:}

That there will be more ADR-2 enzyme in the nucleus than the cytoplasm.

\section{Potential Impact:}

Knowing the location of the edited RNA will allow researchers to have a better idea of what mechanisms influence the editing of ADAR, and what dictates the localization of dsRNA. Current theories involve the number of inosine groups, cellular conditions which effect localization, and nuclear retention molecules other than inosine. 